This item is the archived peer-reviewed author-version of:

\title{
Tunable stress induced magnetic domain configuration in FePt thin films
}

\section{Reference:}

Álvarez N.R., Vázquez Montalbetti M.E., Gómez J.E., Moya Riffo A.E., Vicente Álvarez M.A., Goovaerts Etienne, Butera A..Tunable stress induced magnetic domain configuration in FePt thin films

Journal of physics: D: applied physics - ISSN 0022-3727 - 48:40(2015), p. 1-10

Full text (Publishers DOI): http://dx.doi.org/doi:10.1088/0022-3727/48/40/405003

To cite this reference: http://hdl.handle.net/10067/1312640151162165141 


\title{
Tunable stress induced magnetic domain configuration in FePt thin films
}

\author{
N. R. Álvarez, J. E. Gómez, A. E. Moya Riffo, and M. A. Vicente Álvarez \\ Centro Atómico Bariloche (CNEA) and Conicet, \\ 8400 Bariloche, Río Negro, Argentina. \\ M. E. Vázquez Montalbetti \\ Ingeniería Química, Facultad Regional Villa María, \\ Universidad Tecnológica Nacional, 5900 Córdoba, Argentina. \\ E. Goovaerts \\ Experimental Condensed Matter Physics, \\ Physics Department, University of Antwerp, \\ Universiteitsplein 1, BE-2610 Antwerpen, Belgium.
}

\author{
A. Butera* \\ Centro Atómico Bariloche (CNEA), \\ Instituto Balseiro (U. N. Cuyo), and Conicet, \\ 8400 Bariloche, Río Negro, Argentina.
}

(Dated: March 25, 2015) 


\begin{abstract}
We present a study of the magnetic properties of chemically disordered ferromagnetic $\mathrm{FePt}$ films of $100 \mathrm{~nm}$ thickness that have been grown by sputtering with different Ar pressures (3 mTorr $\leq P_{\text {Ar }} \leq 13$ mTorr). We found that the residual stress can be controlled by the sputtering pressure, which in turn allows to tune the perpendicular magnetic anisotropy due to magnetoelastic effects. Films deposited at lower Ar pressures display an in-plane compressive stress that favors an out of plane component of the magnetization and the formation of a magnetic domain structure in the form of stripes. For higher pressures the stress is relaxed and the magnetic configuration changes to planar domains. These results show the possibility to accurately tune the initial magnetic state in films with potential applications in magnetoelectrically coupled devices.
\end{abstract}

PACS numbers: $75.70 . \mathrm{Kw}, 75.50 . \mathrm{Bb}, 75.70 . \mathrm{Ak}, 75.30 . \mathrm{Gw}, 75.60 . \mathrm{Ch}$

Keywords: FePt, thin films, magnetoelastic effects, stripe domains.

*Electronic address: butera@cab.cnea.gov.ar; Also at INN - Instituto de Nanociencia y Nanotecnología 


\section{INTRODUCTION}

Renewed interest in magnetically soft magnetostrictive Fe alloys (for example FeGa, FeCo, FePt and FePd) appeared recently because of potential applications in magnetoelectrically coupled devices, $[1-4]$ which combine a ferromagnetic film with a piezoelectric substrate in order to control the magnetic anisotropy of the ferromagnet using electric fields. These heterostructures can be used as bistable magnetoelectric memory devices[4] as well as tunable microwave components, such as filters, attenuators, phase-shifters or resonators.[1-3] In particular, FePt has a relatively narrow ferromagnetic absorption line and a considerably large magnetostriction and saturation magnetization. In combination with PMN-PT $\left(\mathrm{PbMg}_{1 / 3} \mathrm{Nb}_{2 / 3} \mathrm{O}_{3}-\mathrm{PbTiO}_{3}\right)$ piezoelectric wafers, magnetoelectric couplings of several tens of Oe.cm $/ \mathrm{kV}$ in the microwave region have been reported.[3] However, the magnetoelectric response was not the same in similar systems (see for example Refs. [3] and [4]), which may be due to the effect of different growing conditions on the initial magnetic state.

FePt alloys of equiatomic composition are formed in an equilibrium thermodynamic phase of tetragonal symmetry, the chemically ordered fct structure called $\mathrm{L} 1_{0}$, consisting of successive layers of Fe and Pt. Their properties have been extensively studied in the last years as a result of the increasing interest in ultrahigh coercivity materials in the magnetic recording industry. On the other hand, FePt thin films deposited at room temperature tend to grow in a chemically-disordered magnetically-soft fcc phase called A1, with potential applications in magnetoelectronics. The magnetic anisotropy and domain structure of these films have been recently investigated[5-12]. It was found that the domain structure depends on the film thickness and it was suggested that the perpendicular anisotropy that originates the stripe domain pattern could be due to residual stress, crystalline texture, or a combination of both effects.

Ferromagnetic thin films can show a magnetic domain structure in the form of parallel stripes above a critical thickness $d_{c r}$ that can be induced by the presence of a perpendicular magnetic anisotropy. In a simplified picture the stripe structure can be thought as state in which the magnetization is essentially in the film plane, but has a relatively small out of plane component with a modulation period that varies approximately (for other parameters

fixed) as the square root of the film thickness. The value of the critical thickness above which the stripe structure is observed depends on the $Q$-factor, the ratio between the 
perpendicular anisotropy $\left(K_{\perp}\right)$, and the demagnetizing energy $\left(2 \pi M_{s}^{2}, M_{s}\right.$ is the saturation magnetization), $Q=K_{\perp} / 2 \pi M_{s}^{2}$, i.e. $d_{c r}=d_{c r}(Q)$. For $Q<1$ the critical thickness above which the stripe structure is observed increases rapidly when $Q$ decreases, [13-17] while for $Q \geq 1$ the magnetization vectors are perpendicular to the film surface and a stripe (or bubble) domain pattern is expected for all film thicknesses. Different films exhibiting this domain configuration are known, and have been characterized both experimentally[5, 18-23] and theoretically[13-17].

It is know that when metals are deposited on an amorphous substrate different textures can result depending on the balance between surface and interface energy during film formation and growth.[24] For fcc metals deposited on naturally oxidized Si at room temperature a [111] texture is generally observed for films thinner than a few hundred nanometers.[24] In physical vapor deposition processes, such as sputtering, the type of residual stress depends strongly on the pressure of the sputtering gas. Low Ar pressures usually give in-plane compressive stresses while above a critical pressure, that is typically in the range of 1-10 mTorr, a change occurs and in-plane tensile stresses are observed.[25, 26] The low-pressure compressive regime is ascribed to the elastic deformation produced during film growth by the energetic Ar ions.[25, 26] At higher pressures particle bombardment is reduced and the crystallization process tends to shrink the film (relatively to the substrate) giving rise to a tensile stress.[26]

As already mentioned, the perpendicular magnetic anisotropy can be induced by magnetoelastic or magnetocrystalline effects. There is a report in FePt thin films[27] showing that the residual stress can be controlled by using different Ar pressures during the film fabrication process, which in turn modifies the $\mathrm{A} 1$ to $\mathrm{L} 1_{0}$ transformation temperature. However, it was also reported[5] that FePt thin films tend to grow with a strong [111] texture (the magnetocrystalline easy axis for the A1 fcc phase) that could induce the appearance of a perpendicular magnetic anisotropy and the formation of stripe magnetic domains.

In order to elucidate the dominant mechanism that gives rise to the effective perpendicular anisotropy and the formation of stripe magnetic domains above a critical thickness, we have grown a series of FePt thin films of a fixed thickness but deposited using different sputtering pressures.

X-ray diffractometry, Energy Dispersive X-ray analysis (EDS) and Rutherford Back Scattering (RBS) were used to determine the lattice parameters, the degree of residual stress and 
the composition of the films as a function of the Ar sputtering pressure. Dc magnetometry, Ferromagnetic Resonance (FMR) and Magnetic Force Microscopy (MFM) were employed for the determination of the magnetic anisotropy and the period of the stripe patterns.

\section{FILM FABRICATION AND STRUCTURAL CHARACTERIZATION}

FePt films of $100 \mathrm{~nm}$ thickness were fabricated by dc magnetron sputtering on naturally oxidized Si (100) substrates using different Ar pressures. The samples were deposited from a $3.8 \mathrm{~cm}$ diameter FePt alloy target with a nominal atomic composition of 50/50. We deposited six films with $P_{\mathrm{Ar}}=3,5,7,9,11$, and 13 mTorr using a sputtering power of 20 W. The deposition rate was checked for $P_{\mathrm{Ar}}=3$ and 9 mTorr giving a similar value of $\sim 0.22$ $\mathrm{nm} / \mathrm{s}$, indicating that with the power used the growth speed is approximately constant in the studied $P_{\text {Ar }}$ range. All samples were covered with a protective Pt layer of approximately $5 \mathrm{~nm}$.

The six films have been characterized by X-ray diffractometry using a conventional BraggBrentano $\theta-2 \theta$ scan, in which $2 \theta$ is the angle between the incident and the diffracted beam. All diffractograms were obtained using $\mathrm{Cu} \mathrm{K}_{\alpha}$ radiation $\left(\lambda_{\mathrm{Cu}}=0.15418 \mathrm{~nm}\right)$. A rocking curve around the $\mathrm{Si}$ (400) reflection of the substrate was previously made to guarantee a correct alignment. In Fig. 1 we observe that in the angular range $20^{\circ} \leq 2 \theta \leq 90^{\circ}$ the (111), (200), (311) and (222) reflections originating from the fcc-A1 FePt phase can be detected (the position of the (220) peak is superimposed with the (400) reflection of the Si substrate and could not be detected). A small peak due to the Pt capping layer is also observed close to $2 \theta \sim 39^{\circ}$. The position of the FePt (111) reflection, which is proportional to the

interplanar distance parallel to the film surface (Bragg's law), shifts to larger angles with increasing Ar pressure, indicating a reduction in $d(111)$. In Fig. 2(a) we show this behavior. Note that the interplanar distance is reduced by approximately $0.5 \%$ between $P_{\mathrm{Ar}}=3$ and 13 mTorr. For the highest Ar pressure the interplanar distance is almost coincident with the value estimated from the X-ray diffraction pattern measured in the sputtering target $\left(d_{0 \text { (bulk })}(111)=0.22025(10) \mathrm{nm}\right)$ and also with data reported in ICDD-PDF[28]. Assuming that this value corresponds to a fully relaxed state, it is then inferred that the films grown at low $P_{\mathrm{Ar}}$ are under an in-plane compressive stress that produces a perpendicular expansive 




FIG. 1: (color online) X-ray diffractograms of the FePt films sputtered using different Ar pressures. In the angular range $20^{\circ} \leq 2 \theta \leq 90^{\circ}$ the (111), (200), (311) and (222) reflections from the A1 FePt phase can be detected. Aside from peaks from the Si substrate, a small diffraction due to the $\mathrm{Pt}$ capping layer (111) reflection is observed close to $2 \theta=39^{\circ}$.

strain. The stress is reduced for increasing pressures and even changes sign for large values of $P_{\mathrm{Ar}}$.

The orientation dependence of the interplanar distances can be analyzed using the $\sin ^{2} \psi$ method.[27] In this technique different series of $\theta-2 \theta$ scans are performed around a diffraction peak for different angular offsets of magnitude $\psi$ and azimuthal angles $\phi$ (the diffraction geometry can be seen in the inset of Fig. 3). This method is often used to estimate the strain in thin films which, in the case of a biaxial strain, can be obtained from the following 




FIG. 2: (color online) (a) Interplanar $d(111)$ distance and (b) Intensity ratio $I_{111} / I_{200}$ as a function of the Ar pressure during sputtering. In panel (a) the bulk $d_{\text {bulk }}(111)$ interplanar distance was obtained from an X-ray diffraction pattern of the sputtering alloy target. The relaxed value of $d(111)$ in films was estimated using the $\sin ^{2} \psi$ method.

simplified expression, [29]

$$
\frac{d(h k l)-d_{0}(h k l)}{d_{0}(h k l)} \approx\left(\varepsilon_{x}-\varepsilon_{z}\right) \sin ^{2} \psi+\varepsilon_{z} \approx-\frac{\nu+1}{2 \nu} \varepsilon_{z} \sin ^{2} \psi+\varepsilon_{z},
$$

that can be written as

$$
d(h k l) \approx-\frac{\nu+1}{2 \nu} \varepsilon_{z} d_{0}(h k l) \sin ^{2} \psi+\left(\varepsilon_{z}+1\right) d_{0}(h k l) .
$$

In the above equations $\varepsilon_{x}$ and $\varepsilon_{z}$ are the in-plane and out of plane strains, $d(h k l)$ and $d_{0}(h k l)$ are the strained and relaxed interplanar distances, and $\nu$ is the Poisson's ratio $(\nu \sim 0.33$ for $\mathrm{FePt}[27,30,31])$. Due to the experimental geometry, the maximum value of $\psi$ must be 
always lower than $\theta$ and for this reason it is convenient to choose a high angle reflection to cover a wide range of $\psi$ values. For the $P_{\mathrm{Ar}}=3$ mTorr sample we selected the (311) reflection because the (222) peak, that would have been a better choice for a direct comparison with the $d(111)$ data of Fig. 2(a), had an intensity that decreased very rapidly for $|\psi|>0$. From the linear fit of the plot of the interplanar spacing $d(311)$ vs. $\sin ^{2} \psi$ (see Fig. 3) it was possible to extract the strain perpendicular to the film plane, $\varepsilon_{z}=(3.89 \pm 0.4) \times 10^{-3}$ and the relaxed value of the (311) interplanar distance in the 3 mTorr film, $d_{0(\text { film })}(311)=0.11518(5) \mathrm{nm}$. This value was used to estimate $d_{0(\text { film })}(111)=\sqrt{11 / 3} d_{0(\text { film })}(311)=0.22055(10) \mathrm{nm}$. Within experimental error, similar results were obtained for three different azimuthal angles $\phi=0^{\circ}$, $45^{\circ}$ and $90^{\circ}$, which indicates that in-plane stresses are almost isotropic. This interplanar spacing is larger than the value found in the sputtering target, the difference probably arising due to the fact that the film is in the $\mathrm{A} 1$ phase while the target is partially in the $\mathrm{L} \mathrm{i}_{0}$ phase. As the $\sin ^{2} \psi$ method is very time consuming and the results do not differ significantly from those obtained from conventional $\theta-2 \theta$ measurements, we adopted $d_{0(\text { film })}(111)=$ $0.22055(10) \mathrm{nm}$ as the relaxed lattice spacing in our films and calculated the strain from the position of the (111) peak in $\theta-2 \theta$ scans and the relation $\varepsilon_{z}=d(111) / d_{0(\text { film })}(111)-1$.

We have also estimated the intensity ratio between the (111) and (200) diffraction peaks (see Fig. 2(b)). A strong [111] texture was found in all the samples. As a reference, $I_{111} / I_{200} \sim 1.4$ for a collection of randomly oriented grains. It is also observed that the [111] texture tends to increase for larger Ar pressures. In the case of fcc metallic films deposited on an amorphous substrate, surface/interface energy minimization is achieved when a [111] texture is developed.[24] However, when the films are stressed, the elastic strain energy density is minimized by a [100] texture if the elastic constants have a Zener anisotropy ratio larger than one, i.e. $A_{Z}=2 C_{44} /\left(C_{11}-C_{12}\right)>1$, which is usually the case for fcc metals. As far as we know, there are no reported experimental values of the elastic constants for the A1 phase of FePt single crystals. There is one experimental work in $\mathrm{L}_{0}$ polycrystalline films[32] that also mentions a range of values $\left(A_{Z} \sim 2-3\right)$ obtained from different theoretical estimations. There are, however, theoretical works $[33,34]$ that ended up with $A_{Z} \lesssim 1$, elastic constants (particularly $C_{12}$ and $C_{44}$ ) that differ at least by a factor of 5 with the experimental values of most common fcc metals and alloys, [35, 36] and a Poisson's ratio $\nu=C_{12} /\left(C_{11}+C_{12}\right) \sim 0.02$, which makes these calculation somewhat unreliable. In the isostructural compound $\mathrm{FePd}$ there are reports[37, 38] on the single crystal elastic constants 
in both phases, with $A_{Z}=3.1$ for the A1 phase and $A_{Z} \sim 2.8$ for the $\mathrm{L} 1_{0}$ structure. The elastic constants $C_{11}, C_{12}$ and $C_{44}$ are very similar in both phases. This last result suggests an anisotropy ratio $A_{Z}>1$ for fcc FePt, but measurements in single crystalline samples are needed to confirm this assumption. In the situation $A_{Z}>1$, strained films are expected to develop a [100] texture in order to reduce the strain energy density while relaxed samples favor the [111] texture. This is consistent with the experimental results presented in Fig. 2(b) that show a decreasing [111] texture for lower Ar pressures.

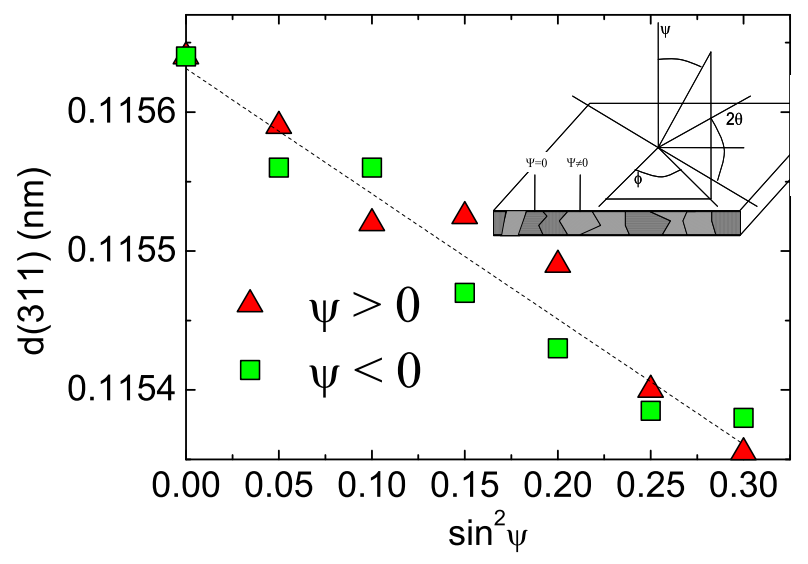

FIG. 3: (color online) Interplanar $d(311)$ distance as a function of the angle $\psi$. From the slope and the ordinate of the fit it is possible to obtain the strain and the relaxed lattice parameter. Data were measured in the sample sputtered at $P_{\mathrm{Ar}}=3 \mathrm{mTorr}$. The inset shows the geometry and angles involved in the $\sin ^{2} \psi$ method.

We also analyzed the FWHM (Full Width at Half Maximum) linewidths $\Delta 2 \theta$ of the (111) and (222) reflections (corrected by the instrumental linewidth) as a function of $P_{\mathrm{Ar}}$ and found that they tend to decrease for larger values of $P_{\mathrm{Ar}}$, as can be seen in Fig. 4 . For the determination of the dominant contribution to the line broadening we compared the width of the (111) and (222) reflections. It is known[39] that the most important sample dependent factors of line broadening in X-ray diffraction experiments are finite crystallite size and microstrain. Anisotropic in-plane stress was not considered due to the results already discussed for the $P_{\mathrm{Ar}}=3 \mathrm{~m}$ Torr film. If $\Delta 2 \theta_{111}$ is assumed to be caused only by 
finite crystallite size of diameter $d_{\text {crist }}$, the Scherrer formula $(K \lesssim 1$ is a dimensionless shape factor), [40]

$$
\Delta 2 \theta=2 / \pi K \lambda_{\mathrm{Cu}} /\left(d_{\text {crist }} \cos \theta\right),
$$

predicts a linewidth for the high angle reflection $\Delta 2 \theta_{222}=\Delta 2 \theta_{111} \cos \theta_{111} / \cos \theta_{222}$, considerably smaller than the observed values, as presented in Fig. 4(b). If, on the other side, microstrains are causing a fluctuation in the interplanar spacing $d$ of magnitude $\Delta d$, the broadening may be estimated[40] from

$$
\Delta 2 \theta \sim 8 / \pi(\Delta d / d) \tan \theta
$$

and results in $\Delta 2 \theta_{222}=\Delta 2 \theta_{111} \tan \theta_{222} / \tan \theta_{111}$, in much better agreement with the experimental values, as can be seen in Fig. 4(b). Due to the low signal to noise ratio of the (222) diffraction peak, we did not separate the $K \alpha_{1}-K \alpha_{2}$ doublet, which, if corrected, would have given a slightly smaller $\Delta 2 \theta_{222}$. From Fig. 4 (b) it can also be deduced that the microstrain values decrease from $\Delta d / d \sim 0.011$ for $P_{\mathrm{Ar}}=3$ mTorr to $\Delta d / d \sim 0.004$ for $P_{\mathrm{Ar}}=13$ mTorr. We also performed a Williamson-Hall[41] treatment to the data in order to separate microstrain from finite size effects. Assuming Lorentzian lineshapes the following relation may be deduced,[41]

$$
(\pi / 2) \Delta 2 \theta \cos \theta=K \lambda_{\mathrm{Cu}} / d_{\text {crist }}+4(\Delta d / d) \sin \theta \text {. }
$$

Using the peak positions and the linewidths of the (111) and (222) reflections we obtained that microstrain is the dominant mechanism of line broadening and that the crystallite size diameter is approximately independent of the sputtering pressure with an average value $<d_{\text {crist }}>=34(9) \mathrm{nm}$.

In order to check if there was a compositional dependence with $P_{\mathrm{Ar}}$ we performed EDS in the whole set of samples. We analyzed two different regions of each sample to account for possible inhomogeneities. After considering the $5 \mathrm{~nm}$ Pt capping layer in the calculation of the relative concentrations, we found out that the film composition is almost independent of $P_{\mathrm{Ar}}$, with the largest $\mathrm{Pt}$ concentration occurring for $P_{\mathrm{Ar}}=9 \mathrm{mTorr}$ As can be deduced from Fig. 4(c)) the average Fe/Pt ratio is $47(2) / 53(2)$. This value is slightly different than the nominal 50/50 composition, but close to the 45/55 ratio measured in the sputtering target. RBS spectrometry data obtained with high energy alpha particles showed also, 
within experimental error, a pressure independent composition and the absence of trapped Ar atoms.

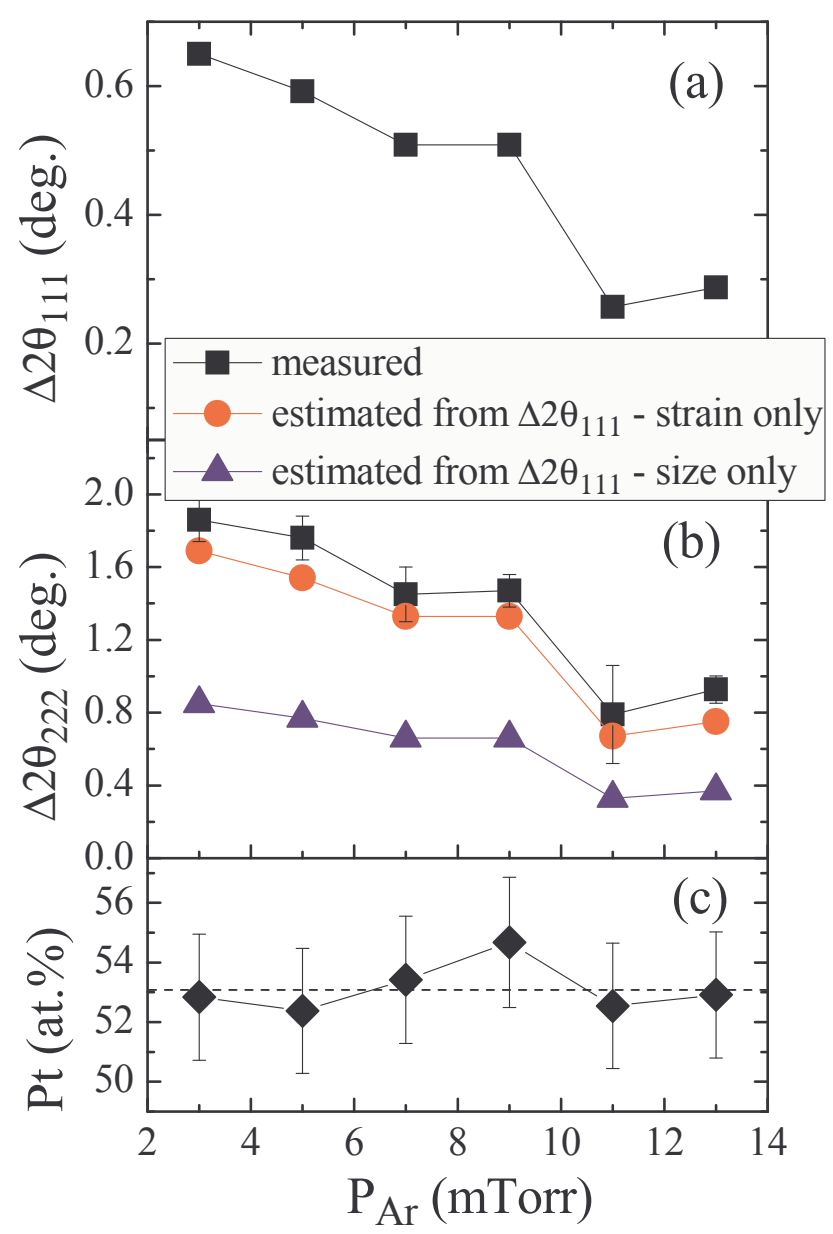

FIG. 4: (color online) Measured FWHM linewidth of (a) the (111) and (b) the (222) reflections as a function of the Ar sputtering pressure. We also plotted the estimated line broadening $\Delta 2 \theta_{222}$ obtained from $\Delta 2 \theta_{111}$ if either crystallite size or microstrain broadening is assumed. Panel (c) shows the atomic concentration of $\mathrm{Pt}$ as a function of $P_{\mathrm{Ar}}$. 


\section{MAGNETIC MEASUREMENTS}

\section{A. dc Magnetization and Magnetic Force Microscopy}

Room temperature magnetization data were measured using a LakeShore model 7300 VSM, capable of a maximum field of $20 \mathrm{kOe}$. For out of plane measurements we also used a Quantum Design SQUID magnetometer in which a maximum field of $50 \mathrm{kOe}$ can be reached. The surface magnetic domain structure of the films was studied by Magnetic Force Microscopy with a Veeco Dimension 3100 AFM/MFM with Nanoscope IV electronics. We used medium moment, medium coercivity magnetic MESP tips from Bruker. As the tip was magnetized along its axis, the force gradient normal to the film plane is detected in all cases. To obtain the magnetic images we used the tapping lift mode with a second scan separation of $30 \mathrm{~nm}$ and phase detection.

It is well know that in thin films there is a close correlation between the magnetization vs. field curves and the domain configuration. In particular, films with stripe domains have characteristic $M$ vs. $H$ in-plane loops in which the following features are often observed:[5, $14,22]$

i) the low field part of the curve increases almost linearly from remanence until the saturation field is reached. This in-plane saturation field was shown to increase with film thickness following approximately[14] the relationship

$$
H_{S \|}=H_{S \perp}\left[1-d_{c r}(Q) /(d \sqrt{1+Q})\right],
$$

where $H_{S \perp}=2 K_{\perp} / M_{s}$ is the saturation field perpendicular to the film plane. We have explicitly emphasized that the critical thickness is dependent on the $Q$-factor. When the stripe structure is lost, planar domains are formed and the loops become square.

ii) When films of different thicknesses are studied, the in-plane coercivity increases abruptly and the remanence decreases considerably above $d_{c r}$ due to the formation of the stripe structure.

iii) The period of the stripe structure depends on the $Q$-factor. An analytical model proposed by Murayama[14] (called Model III in the original paper) results in the following asymptotic expression for the stripe half period in the case of $Q \rightarrow 0$ (valid for $d>d_{c r}$ ),

$$
\frac{\lambda_{s}}{2} \simeq\left[\frac{(2 \pi)^{2} d^{2} A}{2 \pi M_{s}^{2}}\left(1+\frac{1}{Q}\right)\right]^{1 / 4},
$$


where $A$ is the exchange stiffness constant, which for our films was estimated[5] as $A \sim$ $0.6 \times 10^{-6} \mathrm{erg} / \mathrm{cm}$. This model predicts an increase in $\lambda_{s}$ when $Q$ decreases, if the rest of the parameters remain constant.

We have measured the in-plane hysteresis loop of all samples and correlated these measurements with the MFM images acquired in the remanent state $(H=0)$ after saturating the films with a field of $5 \mathrm{kOe}$. In Fig. 5 we observe that for low Ar pressures $(3$ and 5 mTorr) an almost parallel structure of stripe domains is formed. The corresponding hysteresis loops display the $i$ ) and $i i$ ) features described above for films with a stripe domain structure. In the samples fabricated with $P_{\mathrm{Ar}}=7$ and 9 mTorr some changes can be noted. It is observed that the stripes start to loose their parallelism and that the magnetic signal is lower, indicating a reduction in the out of plane component of the magnetization. In these two samples the $M$ vs. $H$ curves tend gradually to a more "square" shape with smaller coercivity and $H_{S \|}$ and a larger remanence. In particular, the sample with $P_{\mathrm{Ar}}=9 \mathrm{mTorr}$ has a magnetization curve very similar to the films fabricated at higher pressures in which planar magnetic domains are formed. This observation indicates that the critical thickness for $P_{\mathrm{Ar}}=9 \mathrm{~m}$ Torr is very close to $d_{c r}=100 \mathrm{~nm}$. As a reference, we had previously found [5] that for $P_{\mathrm{Ar}}=3 \mathrm{~m}$ Torr the critical thickness is $d_{c r} \sim 30 \mathrm{~nm}$.

From the loops and images of Fig. 5 it is possible to extract the saturation magnetization, $M_{s}$, the coercive field, $H_{C}$, the remanent magnetization, $M_{r} / M_{s}$, and the half period of the stripe structure, $\lambda_{s} / 2$, presented in Fig. 6. The period of the stripe structure was determined by performing a 2D Fourier transform of the MFM images. We estimated an average value of $M_{s}=1050(60) \mathrm{emu} / \mathrm{cm}^{3}$, close to the reported value[42] for bulk FePt, $M_{s}(\mathrm{bulk}) \sim 1100$ $\mathrm{emu} / \mathrm{cm}^{3}$. As already mentioned, the transition from striped to planar domains may be inferred from the loop shape, but when the critical thickness is approached it is necessary to have a direct observation of the domain structure in order to confirm the presence or absence of stripes. We have indicated in Fig. 6 the transition region with a shaded band. In Fig $6(\mathrm{~b})$ we observe that $\lambda_{s} / 2$ tends to increase with increasing pressure. This behavior will be discussed later, after performing an estimation of $K_{\perp}$ and the $Q$-factor for the different films.

\section{B. Determination of the perpendicular anisotropy}



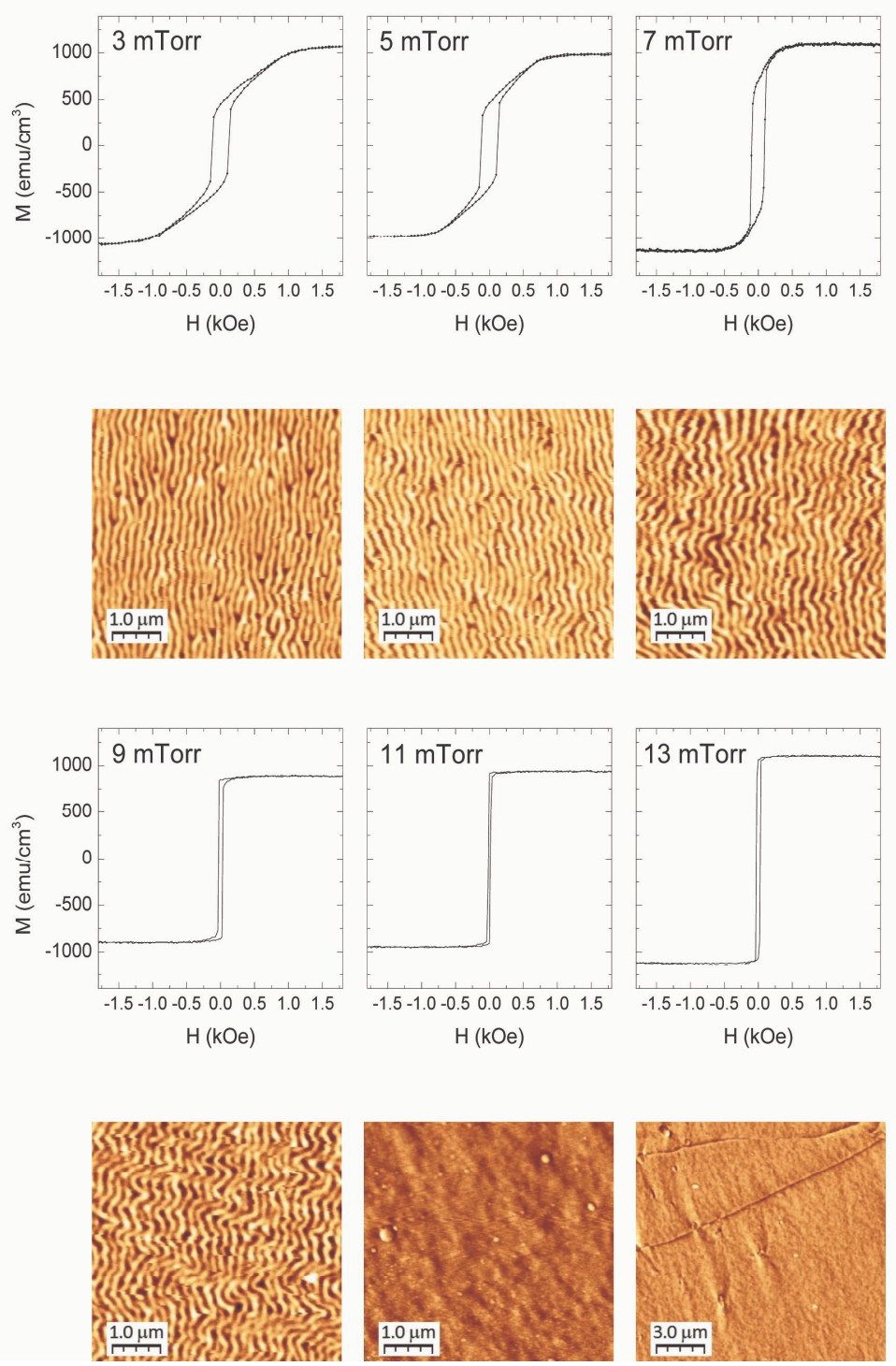

FIG. 5: (color online) dc magnetization loops and corresponding MFM images for the six samples. According to the MFM images the transition from stripe to planar domains occurs between the samples sputtered at 9 and 11 mTorr. For $P_{\text {Ar }} \geq 11$ mTorr the domains are in the film plane and magnetic contrast only appears where domain walls are present.

The perpendicular anisotropy can be determined using different methods. In the presence of a biaxial stress we can use the X-ray diffraction data of Fig. 2 to estimate the magnetoelastic contribution to the anisotropy through the relation[10]

$$
K_{M E}=\frac{3}{2} \lambda E \frac{\varepsilon_{z}}{2 \nu}
$$






FIG. 6: (color online) Coercive field, remanent magnetization (a) and stripe half period (b) as a function of the sputtering Ar pressure. According to the MFM images the transition from striped to planar domains occurs in the shaded region, 9 mTorr $\lesssim P_{\text {Ar }}<11$ mTorr.

where $\lambda$ is the average saturation magnetostriction, $E$ is the Young's modulus, $\varepsilon_{z}=$ $d(111) / d_{0}(111)-1$ is the strain in the direction perpendicular to the film surface, and $\nu$ is the Poisson's ratio. The Young's modulus for FePt at room temperature, $E_{\mathrm{FePt}}=180$ GPa, has been reported by two different authors.[27, 31] However, in those papers it was not mentioned how or where this value was obtained. We employed the vibrating reed technique to measure a small piece of the FePt target and obtained $E_{\mathrm{FePt}}=165(10) \mathrm{GPa}$. This value was corrected because our target was predominantly in the ordered $\mathrm{L}_{0}$ phase and there are reports[37] in the FePd system in which a reduction of the Young's modulus of $20 \%$ in disordered alloys was observed. We then used $E_{\mathrm{FePt}}=130 \mathrm{GPa}$ in the estimation of $K_{M E}$. The reported value $[27,30,31]$ of the Poisson's ratio in FePt is $\nu \sim 0.33$. The saturation 
magnetostriction in $\mathrm{L}_{0}$ materials is known to be positive, but there is a wide range of reported values. In FePt films Aboaf et al.[43] measured $\lambda=70 \times 10^{-6}$ and Spada et al.[30] $\lambda=34 \times 10^{-6}$. There are also recent unpublished differential X-ray absorption spectroscopy results in disordered thin films[44] that give $\lambda_{100} \sim 100 \times 10^{-6}$ and $\lambda_{111} \sim 250 \times 10^{-6}$. In FePd films magnetostriction values range from[45] $\lambda=65 \times 10^{-6}$ to $\lambda=250 \times 10^{-6}$.[46] The value of $K_{M E}$ was estimated using a constant average value $<\lambda>\sim 100 \times 10^{-6}$, which has a considerable uncertainty. In any case, this is only a scale factor and does not affect the dependence of $K_{M E}$ with $P_{\mathrm{Ar}}$. We have plotted $K_{M E}$ as a function of $P_{\mathrm{Ar}}$ in Fig. $7($ a) and also added a right $y$-scale with the $Q$-factor that results assuming $Q=K_{M E} /\left(2 \pi M_{s}^{2}\right)$.

The effective perpendicular anisotropy in thin films can be estimated[47] from the saturation field of the perpendicular magnetization curve $\left(H_{S \perp}\right)$ using the relation $K_{\text {eff }}=H_{S \perp} M_{s} / 2$ and also from the difference between the areas covered by normalized $M$ vs $H$ curves measured with $H$ parallel and perpendicular to the film plane, $A_{\|}-A_{\perp}=H_{S \perp} / 2=K_{\mathrm{eff}} / M_{s}$. From measurements made with $H$ applied in the in-plane and out of plane configurations (see Fig. 8) we have obtained the perpendicular anisotropy values presented in Fig. 7(b) through the relation $K_{\perp}=2 \pi M_{s}^{2}-H_{S \perp} M_{s} / 2$. The relatively large error originates in small sample misalignments and the precise determination of $H_{S \perp}$ due to a nonlinear asymptotic behavior in the saturation region. The area method produced in general somewhat larger anisotropy values which may be due to the non Stoner-Wohlfarth behavior of the loops shown in Figs. 5 and 8 and also to the effects of rotatable anisotropy[5] that have not been considered in the present analysis. In both cases, the perpendicular anisotropy decreases for larger argon pressures, coincident with the results obtained for $K_{M E}$. These findings seem to be in contradiction with the results of Fig. 2(b), that show an increasing [111] texture for larger values of $P_{\mathrm{Ar}}$. As [111] is an easy magnetocrystalline axis, a larger value of the perpendicular anisotropy could be expected for increasing $P_{\mathrm{Ar}}$. However, as can be seen in Fig. 2(b), the [111] texture is already very large for $P_{\mathrm{Ar}}=3 \mathrm{mTorr}\left(\left(I_{111} / I_{200}\right)_{\text {film }} /\left(I_{111} / I_{200}\right)_{\text {random }} \sim 8\right)$ and a further increase in texture contributes only marginally to changes in $K_{\perp}$ due to magnetocrystalline effects.

Another technique that can be used for the determination of the magnetic anisotropy is ferromagnetic resonance. FMR spectra were acquired at room temperature using a commercial Bruker ESP300 spectrometer operating at frequencies $\nu=9.4 \mathrm{GHz}$ (X-band), $24 \mathrm{GHz}$ (K-band) and $34 \mathrm{GHz}$ (Q-band). The maximum field attainable by this equipment is $22 \mathrm{kOe}$. 




FIG. 7: (color online) Magnetoelastic (a) and perpendicular (b) anisotropy as a function of $P_{\mathrm{Ar}}$. Data in the upper panel were estimated from the variation of the interplanar $d(111)$ spacing presented in Fig. 2(a). In the lower panel the anisotropy was calculated from $M$ vs. $H$ loops (VSM) or from ferromagnetic resonance spectroscopy (FMR). Q-factors, indicated on the right axes, are meaningful only for positive values.

W-band $(\nu=94.2 \mathrm{GHz})$ spectra were measured in a Bruker Elexsys E680 system with a 60 $\mathrm{kOe}$ superconducting magnet. The samples were placed in the center of the resonant cavity corresponding to each frequency, where the derivative of the absorbed power with respect to field was measured using a standard field modulation and lock-in detection technique with amplitudes in the range 5-20 Oe. The sample could be rotated inside the resonator in order to measure the resonance field for different orientations. Due to the reduced sample space available in the high frequency resonator, smaller pieces of the same sample were used for 




FIG. 8: (color online) Normalized $M$ vs. $H$ loops measured with the external field applied parallel and perpendicular to the film plane for the six samples. From the difference between the areas covered by both curves it is possible to estimate the effective anisotropy constant (see text).

W-band measurements. We show in Fig. 9 typical FMR spectra for the sample grown using $P_{\mathrm{Ar}}=3$ mTorr, measured with the magnetic field applied parallel and perpendicular to the film plane. One resonance line is generally observed in the in-plane geometry while a few additional absorptions, occurring at fields lower than the main line, can be observed when $H$ is applied parallel to the film normal. These additional modes have been already observed in FePt films and were assigned to the resonance of standing spin waves.[9] A detailed analysis of the behavior of these modes will be published elsewhere.

Using the values of the resonance fields in the parallel and perpendicular configurations $\left(H_{\|}\right.$and $\left.H_{\perp}\right)$, and using the Smit and Beljers [48] formula for the uniform mode of precession, 




FIG. 9: (color online) Typical FMR spectra measured with $H$ parallel (a) and perpendicular (b) to the film plane. Data correspond to the sample sputtered at $P_{\mathrm{Ar}}=3 \mathrm{mTorr}$ and measured at the four available excitation frequencies. Arrows indicate the resonance field of each line. In panel (b) the $Q$-band resonance field was estimated from the extrapolation of the angular variation close to the film normal.

it is possible to arrive to the following equations:

$$
\begin{aligned}
\frac{\omega}{\gamma} & =H_{\perp}-H_{\mathrm{eff}} \\
\left(\frac{\omega}{\gamma}\right)^{2} & =\left(H_{\|}+H_{\mathrm{eff}}\right) H_{\|}
\end{aligned}
$$

with $\omega=2 \pi \nu, \nu$ : excitation frequency; $\gamma=g \mu_{B} / \hbar, g: g-$ factor, $\mu_{B}$ : Bohr magneton; $\hbar$ : reduced Planck constant. $H_{\mathrm{eff}}=4 \pi M_{s}-2 K_{\perp} / M_{s}$ is the effective anisotropy field and should be coincident with the previously defined perpendicular saturation field $H_{S \perp}$. Note 
that these equations are valid only in the case of complete saturation, condition that may not be fulfilled in the parallel geometry for X-band. Also, in some of the samples, the maximum available magnetic field prevented the observation of the perpendicular resonance for Q-band. For these reasons we used K-band and W-band measurements to estimate the $g$-factor obtaining an average value $g=2.07(2)$, and then calculated $H_{\text {eff }}$ using one or both of Eqs. 9. The dependence of $H_{\text {eff }}$ on $P_{\text {Ar }}$ is presented in Fig. 10 where it is observed that $\left\langle H_{\text {eff }}>\right.$, obtained from the average at the four different frequencies, increases with $P_{\mathrm{Ar}}$. This behavior again is consistent with a relaxation of the stress present in the films and the corresponding reduction of the perpendicular anisotropy. The estimated values of $K_{\perp}$ are plotted in Fig. 7 (b) where an excellent agreement was found with the values deduced from dc magnetometry. These values of $K_{\perp}$ are also in very good agreement with the magnetoelastic anisotropy obtained from diffractometry measurements which, as already discussed, is a strong indication that the main contribution to the magnetic anisotropy is due to magnetoelastic effects, which predominate over the average magnetocrystalline anisotropy contribution.

Using the correspondence between $P_{\mathrm{Ar}}$ and the $Q$-factor (or $K_{\perp}$ ) of Fig. $7(\mathrm{~b})$ we can analyze the dependence between the stripe half-period $\lambda_{s} / 2$ and $Q$, as seen in Fig. 11. It is experimentally observed that the period tends to increase for lower values of $Q$, reaching values larger than the film thickness for $P_{\mathrm{Ar}}=9$ mTorr. As far as we know, there are no experimental reports in which the stripe period has been measured as a function of the $Q$-factor in the region $Q<1$. There is one paper on FePt films[21] that shows increasing $\lambda_{s} / 2$ values with increasing $Q$ due to the gradual transformation from the A1 to the $\mathrm{L} 1_{0}$ crystallographic phase. However, in those films the $Q$-values varied in the range $2.5 \leq Q \leq 5$, which is a totally different regime than the one corresponding to our samples. In a relatively simple 1D theoretical model developed in Ref. 15, which only allows the magnetization vector to rotate around the $z$-direction in a single plane, a constant period $\lambda_{s} / 2 \sim d$ is predicted in the limiting case $d \gg \sqrt{A /\left(2 \pi M_{s}^{2}\right)}$. In our samples the film thickness $d$ is much larger than the exchange length $\left(l_{e}=\sqrt{A /\left(2 \pi M_{s}^{2}\right)} \sim 3 \mathrm{~nm}\right)$, so that a stripe half-period close to the film thickness is expected and indeed observed. But the domain structure is evidently more complex than a simple pattern of perfectly parallel stripes, as can be seen in Fig. 5. As already discussed, Model III of Ref. 14 considers an additional degree of freedom for the magnetization angle, which results in a nonconstant functional dependence 




FIG. 10: (color online) Dependence of the effective anisotropy field with the Ar sputtering pressure for the four different FMR frequencies. Full squares indicate the average value of $H_{\text {eff }}$ for each sample. The dotted line corresponds to $4 \pi M_{s}$, the shape anisotropy field for a thin film.

between $\lambda_{s} / 2$ and $Q$. We have plotted in Fig. 11 the behavior predicted by Eq. 7, which is the asymptotic behavior of $\lambda_{s} / 2$ valid for very low values of $Q$. Note that although Eq. 7 has no free parameters (we used the experimentally determined values of $M_{s}, A$, and $d$ ) a reasonably good agreement is found between model and experiments, both in magnitude and in the dependence of $\lambda_{s} / 2$ on $Q$.

Data presented in this work have been measured in films of the same thickness. In order to verify the model of Ref. 14 it is desirable to determine the critical thickness $d_{c r}$ as a function of both the film thickness and the $Q$-factor. We are currently focusing our efforts in this direction, working in the characterization of different sets of samples in which both 
variables are systematically changed.

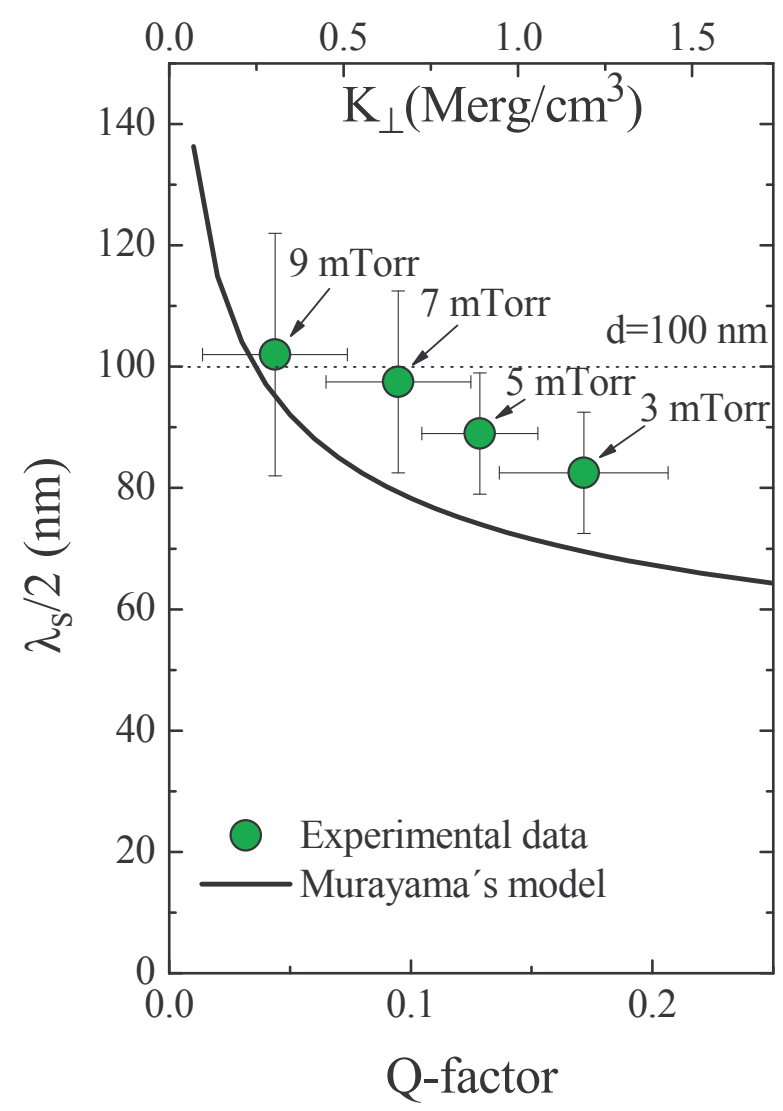

FIG. 11: (color online) Stripe half period as a function of the $Q$-factor (or the perpendicular anisotropy). Full circles correspond to the experimental data while the continuous line was obtained from Murayamas model (Eq. 7). The dashed line indicates the film thickness.

\section{CONCLUSIONS}

Using different experimental techniques we have been able to show that the magnetic domain configuration in FePt ferromagnetic films of a fixed thickness is mainly determined by the degree of residual stresses. This variable was tuned in the present study by the Ar pressure used in the fabrication process. It can be as well externally controlled using, for example, a piezoelectric substrate that can be strained by an applied voltage. The variation 
of the stripe period with the $Q$-factor is reasonably well described by existing theoretical models, which contain no free parameters. The ability to tune the magnetic anisotropy and the domain configuration, together with a deep understanding of the underlying mechanisms, are of capital importance for the design and fabrication of novel magnetoelectric microwave components.

This work was supported in part by Conicet under Grant PIP 112-201101-00482, ANPCyT Grant PICT 2010-0773, and U.N. Cuyo Grant 06/C421, all from Argentina. Financial support through the international cooperation project between Argentina and Belgium MINCyT-FWO FW/11/04 was used in this research. Investment funds for advanced electron-paramagnetic resonance instrumentation from the Flemish Hercules foundation in project AUHA013 are kindly acknowledged. Technical support from Rubén E. Benavides and Matías Guillén is greatly acknowledged. We also thank Dr. Gabriela Alejandro for a critical reading of the manuscript.

[1] T. Fitchorov, Y. Chen, B. Hu, S. Gillette, A. Geiler, C. Vittoria, and V. Harris, J. Appl. Phys. 110, 123916 (2011).

[2] S. Li, H. Du, Q. Xue, X. Gao, Y. Zhang, W. Shao, T. Nan, Z. Zhou, and N. X. Sun, J. Appl. Phys. 115, 17C723 (2014).

[3] J. Vargas and J. Gómez, APL Materials 2, 106105 (2014).

[4] Y. T. Yang, Y. Q. Song, D. H. Wang, J. L. Gao, L. Y. Lv, Q. Q. Cao, and Y. W. Du, J. Appl. Phys. 115, 024903 (2014).

[5] E. Sallica Leva, R. C. Valente, F. Martínez Tabares, M. Vásquez Mansilla, S. Roshdestwensky, and A. Butera, Phys. Rev. B 82, 144410 (2010).

[6] M. Vásquez Mansilla, J. Gómez, and A. Butera, IEEE Trans. Magn. 44, 2883 (2008).

[7] M. Vásquez Mansilla, J. Gómez, E. Sallica Leva, F. Castillo Gamarra, A. Asenjo Barahona, and A. Butera, J. Magn. Magn. Mat. 321, 2941 (2009).

[8] E. Burgos, E. Sallica Leva, J. Gómez, F. Martínez Tabares, M. Vásquez Mansilla, and A. Butera, Phys. Rev. B 83, 174417 (2011). 
[9] D. M. Jacobi, E. Sallica Leva, N. Álvarez, M. Vásquez Mansilla, J. Gómez and A. Butera, J. Appl. Phys., 111, 033911 (2012).

[10] J. M. Guzmán, N. Álvarez, H. R. Salva, M. Vásquez Mansilla, J. Gómez, and A. Butera, J. Magn. Magn. Mat. 347, 61 (2013).

[11] N. Álvarez, G. Alejandro, J. Gómez, E. Goovaerts, and A. Butera. J. Phys. D: Appl Phys. 46, 505001 (2013).

[12] N. Álvarez, E. Sallica Leva, R. C. Valente, M. Vásquez Mansilla, J. Gómez, J. Milano, and A. Butera. J. Appl. Phys. 115, 083907 (2014).

[13] C. Kooy and U. Enz, Philips Res. Rep. 15, 7(1960).

[14] Y. Murayama, J. Phys. Soc. Jap. 21, 2253 (1966).

[15] A. L. Sukstanskii and K. I. Primak, J. Magn. Magn. Mat. 169, 31 (1997).

[16] R. Bručas, H. Hafermann, M. I. Katsnelson, I. L. Soroka, O. Eriksson, and B. Hjörvarsson, Phys. Rev. B, 69, 064411 (2004).

[17] A. Hubert and R. Schäfer, Magnetic Domains, Springer, 3rd printing (2009).

[18] N. Saito, H. Fujiwara, and Y. Sugita, J. Phys. Soc. Japan, 19, 421 (1964).

[19] M. Hehn, S. Padovani, K. Ounadjela, and J. P. Bucher, Phys. Rev. B 54, 3428 (1996).

[20] V. Gehanno, R. Hoffmann, Y. Samson, A. Marty, and S. Auffret, Eur. Phys. J. B 10, 457 (1999).

[21] S. Okamoto, N. Kikuchi, O. Kitakami, T. Miyazaki, Y. Shimada, and K. Fukamichi. Phys. Rev. B 66, 024413(2002).

[22] M. Barturen, B. Rache Salles, P. Schio, J. Milano, A. Butera, S. Bustingorry, C. Ramos, A. J. A. de Oliveira, M. Eddrief, E. Lacaze, F. Gendron, V. H. Etgens, and M. Marangolo, Appl. Phys. Lett. 101, 092404 (2012).

[23] C.A. Ramos, E. Vassallo Brigneti, J. Gómez, and A. Butera, Physica B 404, 2784 (2009).

[24] C. V. Thompson and R. Carel, Mater. Sci. Eng. B, 32, 211 (1995).

[25] C. V. Thompson and R. Carel, J. Mech. Phys. Solids, 44, 657 (1996).

[26] S. Tamulevičius, Vacuum, 51, 127 (1998).

[27] S. N. Hsiao, F. T. Yuan, H. W. Chang, H. W. Huang, S. K. Chen, and H. Y. Lee, Apll. Phys. Lett. 94, 232505 (2009).

[28] ICDD-PDF reference code 00-029-0718.

[29] Residual Stress Measurement by Diffraction and Interpretation, I. C. Noyan and J. B. Cohen, 
Springer-Verlag, New York (1987).

[30] F.E. Spada, F.T. Parker, C.L. Platt, and J.K. Howard, J. Appl. Phys 94, 5123 (2003).

[31] P. Rasmussen, X. Rui, and J.E. Shield, Appl. Phys. Lett. 86191915 (2005).

[32] N. Nakamura, A. Uranishi, M. Wakita, H. Ogi, M. Hirao, and M. Nishiyama, Appl. Phys. Lett. 98, 101911 (2011).

[33] M. Imaizumi, C. A. Soufen, C. A. F. Pintão, L. C. Varanda, and M. Jafelicci Jr., Materials Science and Engineering A, 521-522, 167 (2009).

[34] N. Zotov and A. Ludwig, Intermetallics 16, 113 (2008).

[35] T. Çă̆in, G. Dereli, M. Uludoğan, and M. Tomak, Phys. Rev. B, 59, 3468 (1999).

[36] H. M. Ledbetter and R. P. Reed, J. Phys. Chem. Ref. Data, 2, 531 (1973).

[37] T. Ichitsubo and K. Tanaka, J. Appl. Phys. 96, 6220 (2004).

[38] A. Al-Ghaferi, P. Müllner, H. Heinrich, G. Kostorz, and J. M. K. Wiezorek, Acta Materiala, $54,881(2006)$.

[39] A. R. Bushroa, R. G. Rahbari, H. H. Masjuki, and M. R. Muhamad, Vacuum, 86, 1107 (2012).

[40] Th. de Keijser, E. J. Mittemeijer, and H. C. F. Rozendaal, J. Appl. Cryst. 16, 309 (1983). The factor $2 / \pi$ arises from the proportionality between $\triangle 2 \theta_{F W H M}$ and the integral breadth in a Lorentzian line. For a Gaussian line shape the factor is $\sqrt{4 \ln 2 / \pi}$.

[41] G. K. Williamson and W. H. Hall, Acta Metall. 1, 22 (1953).

[42] H. Kanazawa, G. Lauhoff, and T. Suzuki, J. Appl. Phys., 87, 6143 (2000).

[43] J.A. Aboaf, T.R. McGuire, S.R. Herd, E. Klokholm, IEEE Trans. Mag. 20,1642 (1984) .

[44] M. P. Ruffoni, C.B. Ndao, S. Pascarelli, and N.M. Dempsey, Direct measurement of the local, atomic-scale, Joule magnetostriction of FePt. Joint European Magnetic Symposia, Dublin (2008). Unpublished.

[45] H. Shima, K. Oikawa, A. Fujita, K. Fukamichi, K. Ishida, J. Magn. Magn. Mat. 272-276 $2173(2004)$.

[46] W.Wunderlich, K. Takahashi, D. Kubo, Y. Matsumara, Y. Nishi, J. Alloys Comp. 475339 (2009).

[47] Ultrathin Magnetic Structures I, J.A.C. Bland and B. Heinrich (Eds.), Springer-Verlag, Berlin (2005). p. 75.

[48] J. Smit and H. G. Beljers, Philips Res. Rep. 10, 113 (1955) . 












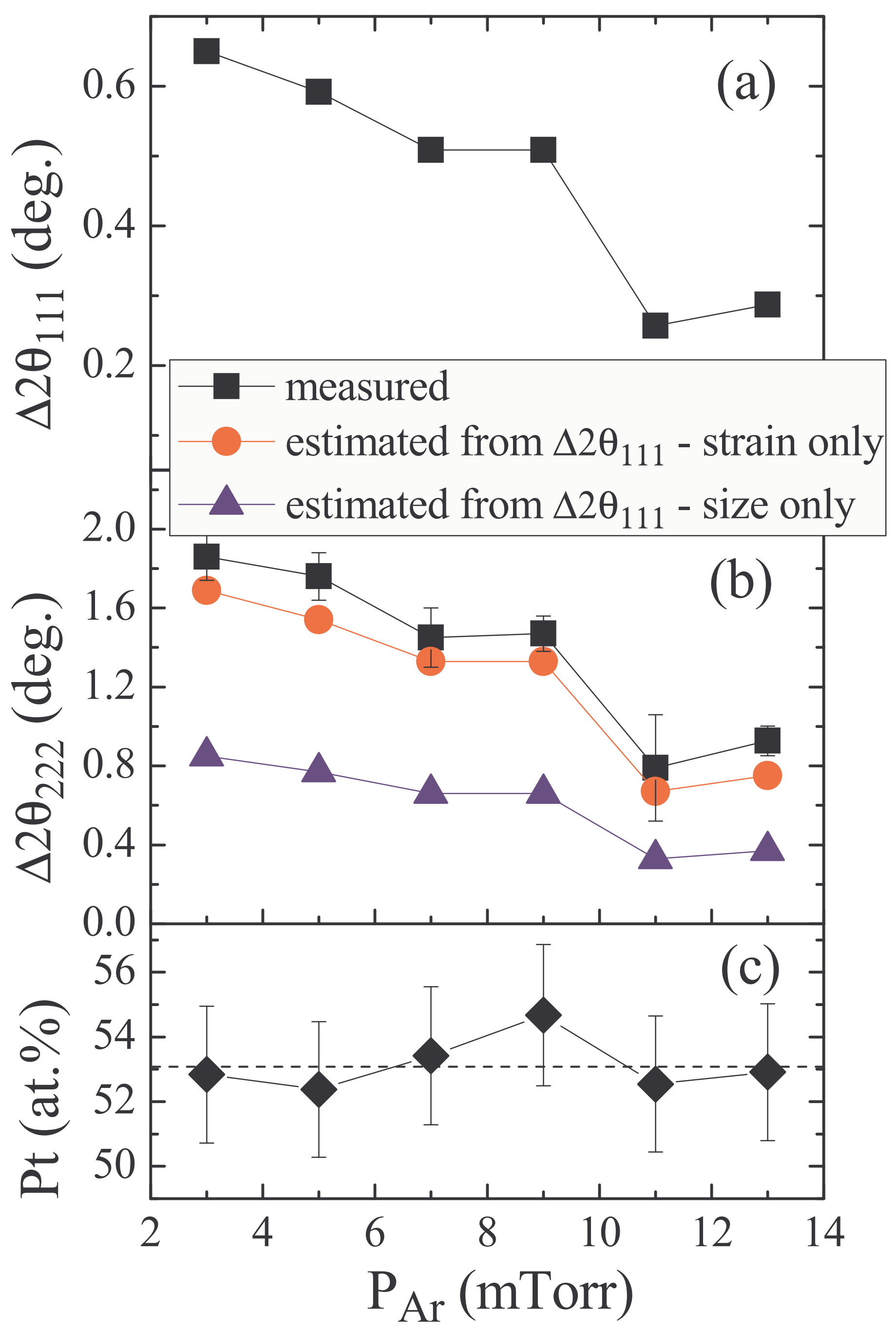



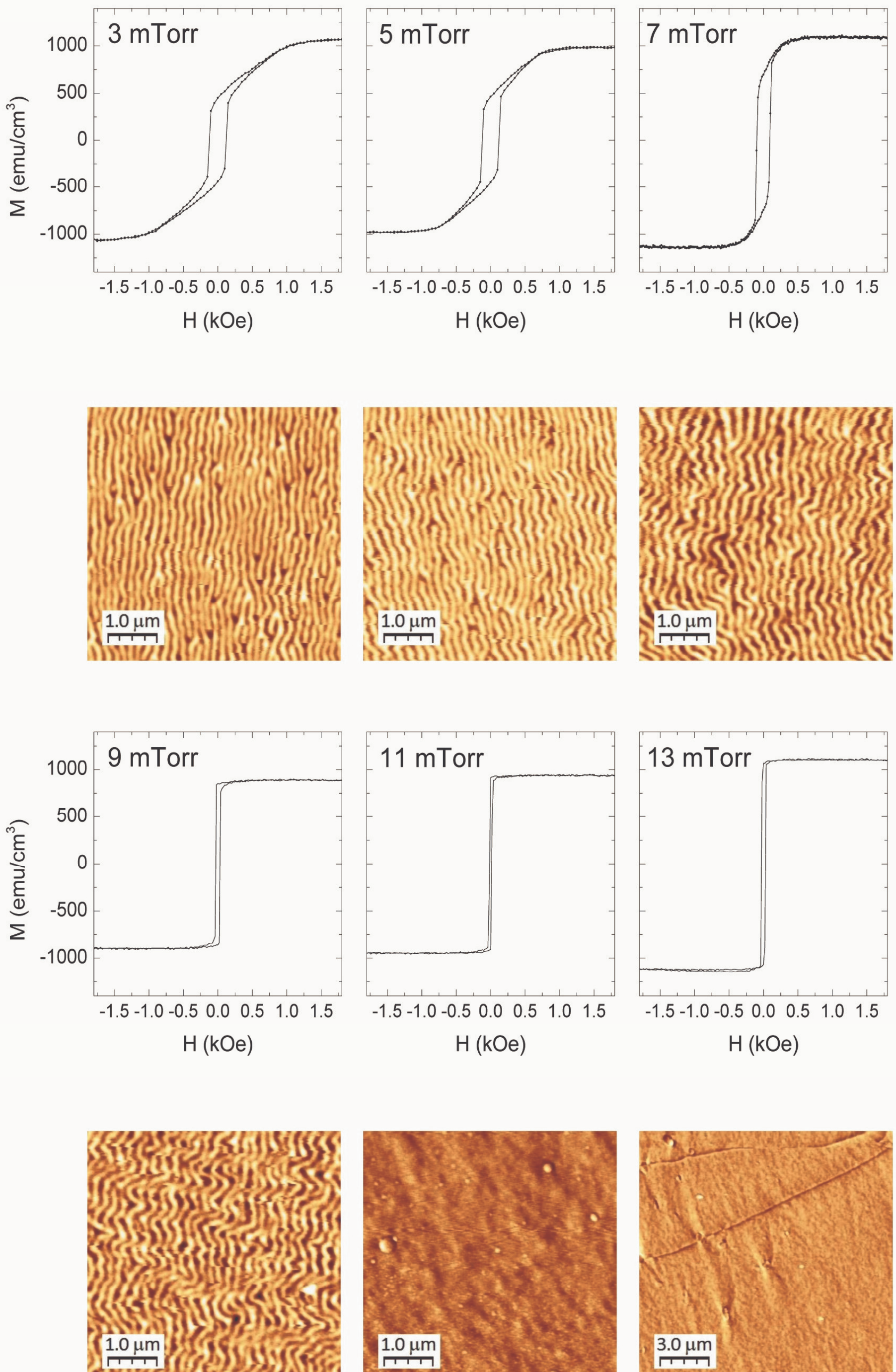


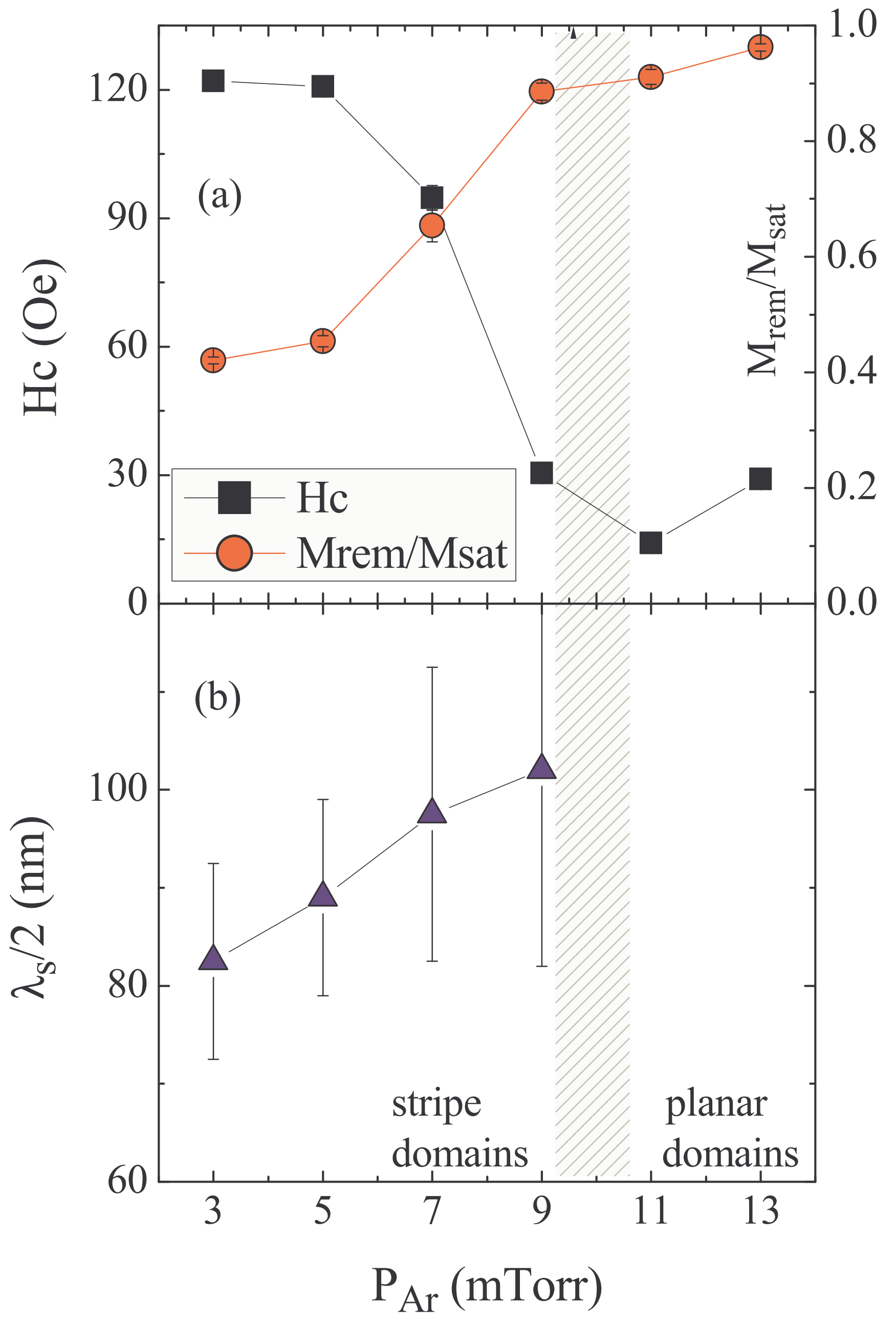












FePt/Pt 3 mTorr

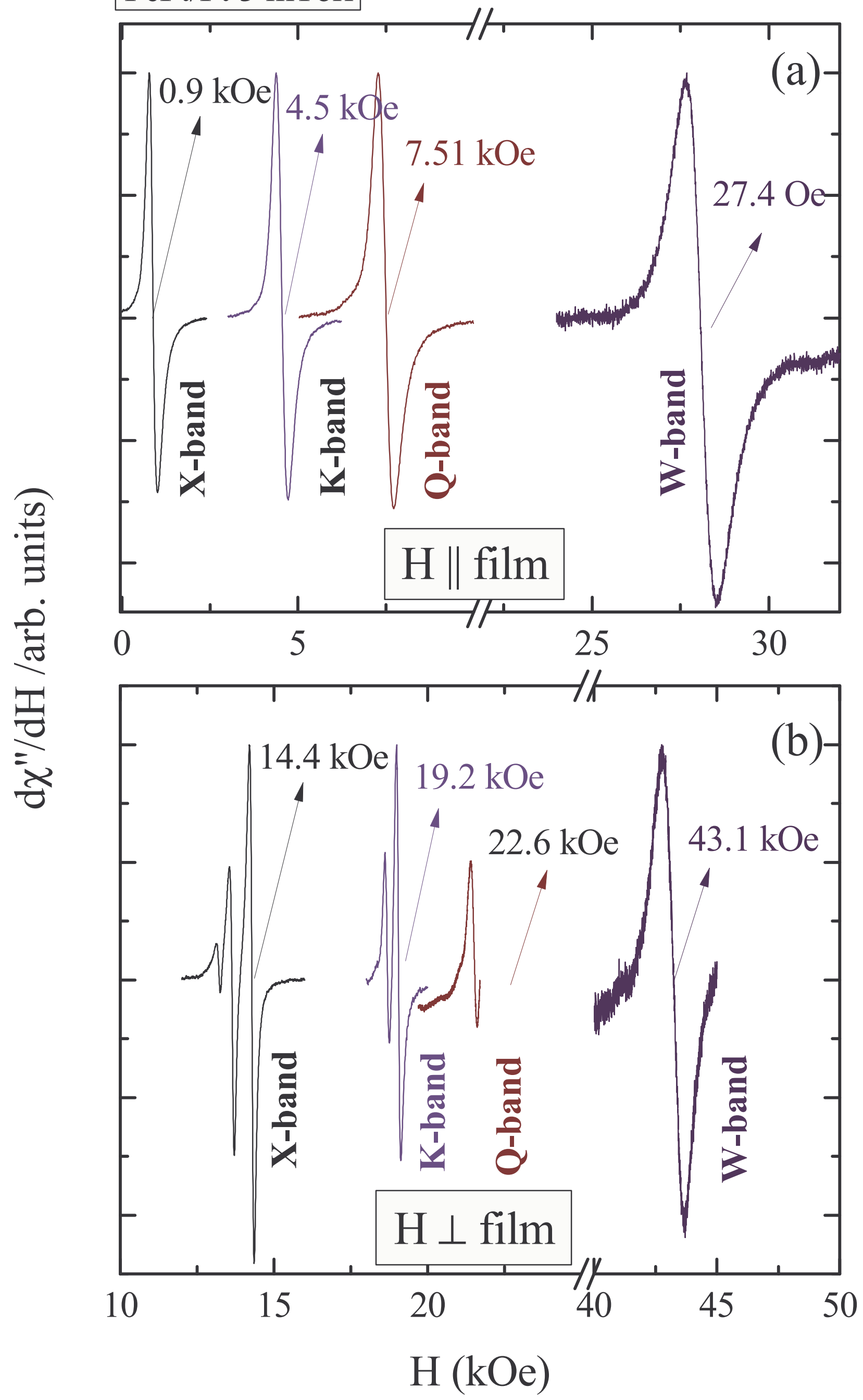







